\title{
Designing a Hollow Langevin Transducer for Ultrasonic Coring
}

\author{
P. Harkness ${ }^{1, a}$, A. Cardoni ${ }^{1, b}$, J. Russell ${ }^{1, c}$ and M. Lucas ${ }^{1, d}$ \\ ${ }^{1}$ School of Engineering, University of Glasgow, Glasgow, G12 8QQ, UK \\ ap.harkness@eng.gla.ac.uk, ba.cardoni@eng.gla.ac.uk

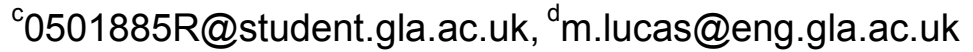

Keywords: Bolted Langevin Transducer, Effect of Preload, Response Functions

\begin{abstract}
A number of architectures for a hollow Langevin ultrasonic transducer are proposed and evaluated. One of these is optimised by finite element modelling and is then manufactured and analysed experimentally. The preload on the transducer ceramics is increased and the effect on the performance is measured. At maximum preload the results of an experimental modal analysis are used to determine the natural frequency and response of both the operating longitudinal mode and unwanted bending modes. The performance of the hollow transducer is compared to a solid commercial transducer containing the same volume of piezoceramic material. The efficiency is shown to be comparable. Higher ultrasonic displacement amplitudes are achieved with the hollow transducer although a lower Q-factor is found.
\end{abstract}

\section{Introduction}

Bolted Langevin transducers are solid structures, with a central bolt running through the centre of a stack of piezoceramic rings and securing the end-masses. The bolt delivers the prestress required to keep the piezoceramics in a state of compression throughout the vibrational cycle. However, for some applications such as rock coring, or when the transducer requires extensive cooling, access through the centre of the transducer may be required.

For example, ultrasonic tools have been suggested as a mechanism by which rock cores can be taken from the subsurface by planetary landers [1]. However, these designs all suffer from the requirement that the ultrasonic apparatus be removed to extract the sample. A hollow transducer, horn and cutting assembly could obviate this need [2]. Additionally, heavy-duty ultrasonic systems often require fan cooling systems [3]. Permitting passage of the coolant through the centre of the stack would increase the surface area available for heat transfer.

\section{Architecture of the Hollow Transducer}

Design procedure. The traditional bolted Langevin transducer (BLT) has a solid central bolt which must be replaced by a hollow structure. There are two options: either the bolt can be moved to the outside of the structure, as a cylinder or as a ring of peripheral columns, or the bolt can be retained inside the transducer but made hollow. Two alternative architectures, a cage and a hollow-bolt, are therefore explored and compared by considerations such as application of prestress, part-count, cost and ease of final assembly. The hollow-bolt architecture is shown to be superior, although the problems associated with an inherently low-stiffness device are recognised. Using different materials for the front and end-masses (steel and aluminium) to maximise unidirectional vibration output, and with reference to the fatigue life of the thin-walled titanium bolt, a prototype of this device is designed using finite element (FE) analysis.

The transducer is manufactured, an experimental modal analysis is conducted using 3D laser Doppler vibrometry, and the extracted modes are compared (in terms of mode shape and natural frequency) to the numerical predictions. Impedance analysis is also carried out and, as the preload in the system is progressively increased, the frequency of the response of the operating longitudinal mode tends towards the modelled predictions. When sufficient preload to stabilise the operating 
frequency is reached a response spectrum is measured and the Q-factor of the transducer is calculated. Further addition of preload is then shown to increase the magnitude and quality factor of the response at the operating frequency. Finally, the hollow transducer is contrasted with a traditional transducer of similar size, to determine its performance with reference to its solid competitors.

External bolting. An external cylinder is unlikely to be practical because it would impede access to the piezoceramics. It would also have to be, effectively, a thin-walled shell. Thin-walled structures, which are inherently non-stiff, are liable to exhibit detrimental transverse modes at ultrasonic frequencies. Furthermore, the structure would preclude the use of all but the thinnest screwthreads for prestressing purposes, and would be extremely vulnerable to fatigue.

The alternative external-bolting approach, consisting of a series of columns around the circumference of the transducer, permits access to the ceramics but would require that the ceramics themselves, which are commercially produced as rings, be shaped to fit around the columns. The number of columns and non-standard ceramics would be difficult, slow and expensive to both manufacture and assemble. The stress applied through each bolt would also have to be equalized to ensure even compressive load on the ceramic stack, as uneven prestress is known to be associated with performance inefficiencies [4].

Internal bolting. Modifying the central bolt, such that it becomes a hollow structure, appears to be a more attractive approach because the walls of the bolt remain sufficiently thick to accommodate screwthreads for assembly purposes. It also permits the use of standard piezoceramic discs and facilitates the even distribution of prestress across the piezoceramic stack. In the absence of other factors, this architecture is preferred for the targeted coring application.

\section{Design of the Hollow Transducer}

In common with several conventional transducers, end masses of steel and aluminium are used in this design to maximise longitudinal vibration output by taking advantage of their different stiffness-to-density ratios. A $90 \mathrm{Ti} / 6 \mathrm{~V} / 4 \mathrm{Al}$ bolt is used because of its high quality factor and fatigue resistance, and PZT-8 ceramics driven by copper electrodes provide the vibration excitation.

The thickness of the bolt wall is set at $3 \mathrm{~mm}$, and the volume of PZT-8 is required to be close to $5 \times 10^{-5} \mathrm{~m}^{3}$ to match the volume of ceramic contained in a commercial transducer known to be effective for rock-coring operations. A separation of $2 \mathrm{~mm}$ is maintained between the central bolt and the electrodes and the interstitial space is filled with dielectric material to prevent internal shorts. A preload screw is provided at one end for final assembly and the other is threaded to accept a half-inch UNF stud. Although a system utilising a hollow transducer would be hollow throughout, the transducer is to be further tested using a hollow horn designed to connect to the commercial transducer and so the central stud connection is required.

The design frequency of the hollow transducer is $20 \mathrm{kHz}$ for operation in the first longitudinal mode. To achieve this, an FE model of the hollow transducer is created in Abaqus, with reference to the fixed design parameters described above. The non-fixed parameters, such as the lengths of the end-masses, are then adjusted to tune the operating frequency. Finally the oscillatory stress in the central bolt is evaluated, assuming a peak-to-peak displacement of the transducer of $10 \mu \mathrm{m}$ and is found to be well below the fatigue limit. The resulting design is presented in Fig. 1. 


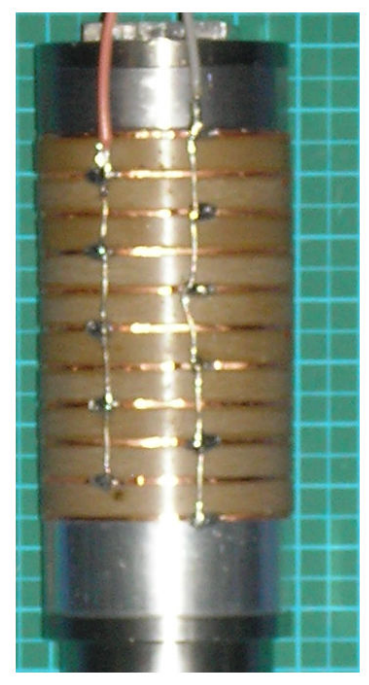

(a)

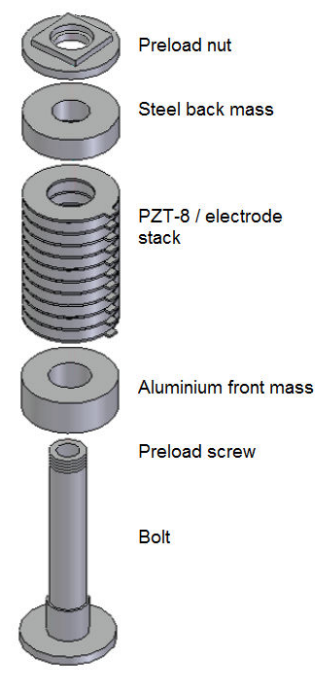

(b)

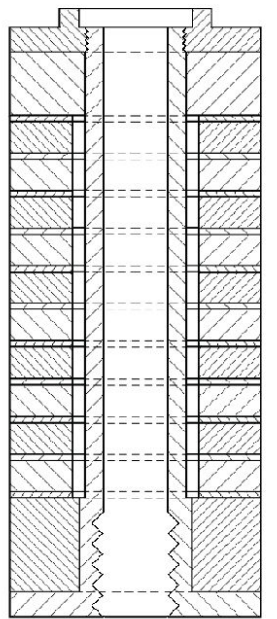

(c)

Figure 1. The hollow transducer, (a) as manufactured, (b) in exploded and (c) in assembled form. The grid spacing in the photograph is $5 \mathrm{~mm}$ and the external wiring to the electrodes is apparent.

\section{Application of Preload}

Clamps are offered up to flats ground on the bolt, front mass and back mass (see Fig. 2(a)), whilst the preload nut is tightened to increase the preload on the stack. This allows preload to be applied to the stack without any torsion which could damage the electrodes. Whilst the actual preload is almost impossible to predict due to the complexities of the transducer structure [5,6], it can be found in practice by measuring the charge on the piezoceramics during the prestressing process [7]. It is known that increasing the preload will tend to increase the first longitudinal resonance frequency of a bolted Langevin transducer as well as the associated Q-factor, and it is reasonable to expect that the same will be true for hollow transducers.

The preload is increased from the minimum required for solid assembly (bias 1) to the point at which no further changes in the frequency of the first longitudinal mode appear to be achievable (bias 4). To measure the prestress applied in this condition, a sample of PZT-8 was subjected to compression testing. After ten seconds at a range of applied pressures, the voltage across the faces was recorded to form a look-up table which was then used to deduce the preload in the transducer after tightening. The minimum preload required to stabilise the frequency behaviour of the device was found to be $10 \mathrm{MPa}$.

\section{Broadband Results}

Fig. 2(b) shows the impedance analysis, and the progression from bias 1 to bias 4 shows how the results of the repeated impedance analyses are affected by increasing preload.

An experimental modal analysis of the transducer is performed at bias 1 using a 3D laser Doppler vibrometer, where the responsive modes, marked A, B, C and D in Fig. 3(a), are extracted during excitation by a white noise signal between 0 and $40 \mathrm{kHz}$. The frequencies of these modes, which are respectively first bending, second bending coupled with first longitudinal, third bending and first radial, are indicated on the frequency response function (FRF) shown in Fig. 3(a). Fig. 3(b) shows a result of the second experimental modal analysis, where a much higher preload (bias 4 ) has been applied. The responsive modes, which are first bending, second bending, first longitudinal and third bending and are marked E, F, G and $\mathrm{H}$ respectively in the figure, are extracted. 


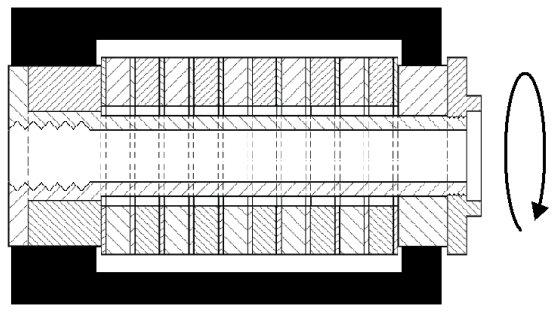

(a)

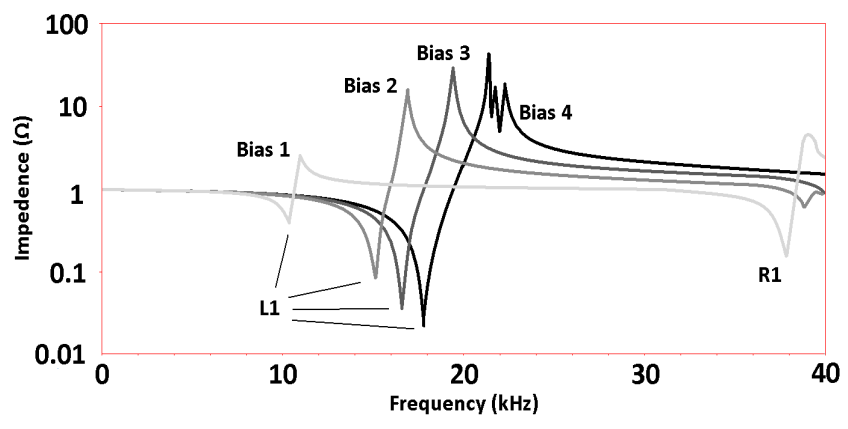

(b)

Figure 2. Clamps are applied (a) such that torque does not reach the PZT as preload is increased, and (b), the measured impedance of the transducer as the preload is increased from bias 1 to bias 4 . L1 peaks refer to the first longitudinal mode and R1 peaks to the first radial mode.

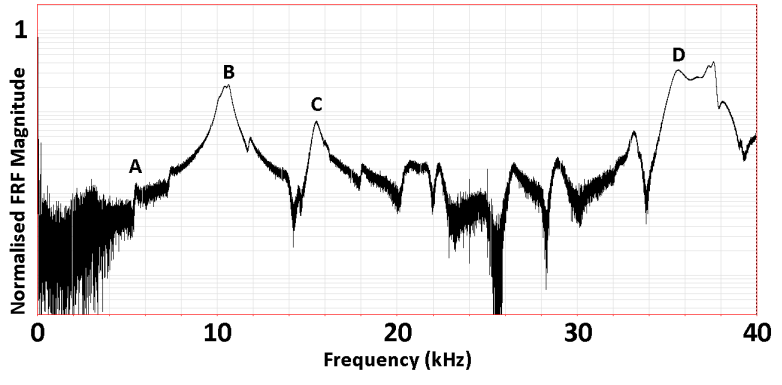

(a)

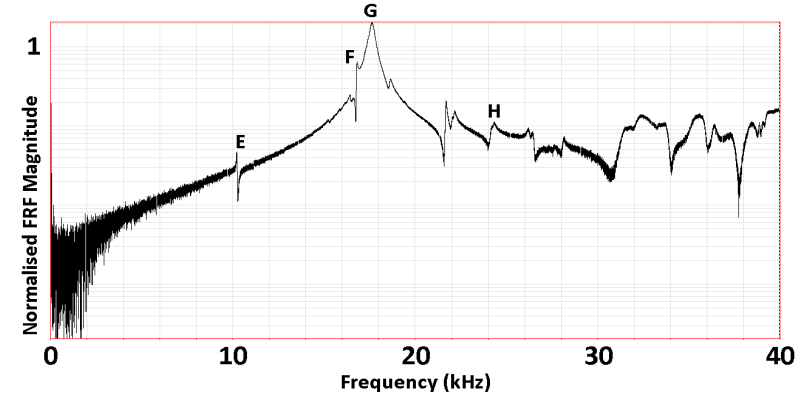

(b)

Figure 3. Normalised longitudinal FRF magnitude at (a) bias 1 and (b) bias 4.

Initially, the operating mode (first longitudinal) of the transducer is excited at $10.3 \mathrm{kHz}$ and is coupled with the second bending mode. Increasing the preload causes the operating mode to decouple from the bending mode, increase in strength by almost an order of magnitude and move towards the $20 \mathrm{kHz}$ value predicted by the finite element model. This final trend is consistent with the behaviour of conventional bolted Langevin transducers [8].

\begin{tabular}{|l|l|l|l|l|l|l|}
\hline & \multicolumn{7}{|c|}{ Modal Frequencies [kHz] } \\
\hline & Bias 1 & Bias 2 & Bias 3 & Bias 4 & FE Prediction \\
\hline $1^{\text {st }}$ Longitudinal & $10.3(52 \%)$ & $15.2(77 \%)$ & $16.6(84 \%)$ & $17.8(90 \%)$ & 19.8 \\
\hline $1^{\text {st }}$ Bending & $5.6(51 \%)$ & - & - & $10.2(94 \%)$ & 10.9 \\
\hline $2^{\text {nd }}$ Bending & $10.3(56 \%)$ & - & - & $16.8(92 \%)$ & 18.3 \\
\hline $3^{\text {rd }}$ Bending & $15.5(57 \%)$ & - & - & $24.2 \quad(90 \%)$ & 27.2 \\
\hline
\end{tabular}

Table 1. Modal frequencies measured at different bias levels. Values in parentheses are percentages with respect to the predicted frequency.

Increasing prestress also causes the frequencies of the radial and bending modes to increase, due to the stiffening of the structure as microscopic voids between the elements are progressively removed. This process can take place due to cold flow of the soft copper electrodes into surface imperfections [9]. As prestress increases from bias 1 to bias 4, the modal frequencies approach the values predicted by the finite element model, as shown in Table 1. However, increasing prestress beyond $10 \mathrm{MPa}$ has a negligible effect on the frequencies of the modes studied. 


\section{Modal Analysis}

The mode shapes, as predicted by FE and measured by EMA are presented in Table. The transducer was self-excited by a random signal.

\begin{tabular}{|l|l|l|l|l|l|}
\hline Mode & EMA & FE & Mode & EMA & FE \\
\hline $1^{\text {st }}$ Longitudinal & & & $2^{\text {nd }}$ Bending & & \\
\hline $1^{\text {st }}$ Bending & & & & & \\
\hline
\end{tabular}

Table 2. Mode shapes measured by EMA and predicted by FE analysis.

\section{Improvements to the Design}

The process of preloading the transducer demonstrated that the application of significant preload requires very considerable torque. The $10 \mathrm{MPa}$ loading needed to stabilise the transducer required approximately $50 \mathrm{Nm}$ and so the preload nut was redesigned to facilitate the use of a hexagonal socket.

FE analysis indicated that this new nut has a negligible influence on the natural frequency of the transducer, but it permitted the preload on the piezoceramic stack to be increased to $20 \mathrm{MPa}$. Although some sources recommend preloads as high as $40 \mathrm{MPa}$ [10], the thin-walled structure of the central bolt makes high loads difficult to apply safely.

\section{Narrowband (Operating Mode) Results}

Fig. 4(a) shows the response of the longitudinal operating mode $(+/-2 \mathrm{kHz}$ range) in bias condition 4 , compared to the response of a solid commercial transducer containing the same volume of piezoceramic. In both cases a sinusoidal excitation of $40 \mathrm{~V}$ (peak-to-peak) has been applied. Fig. 4(b) shows the same measurements after the new nut has permitted doubling of the applied preload.

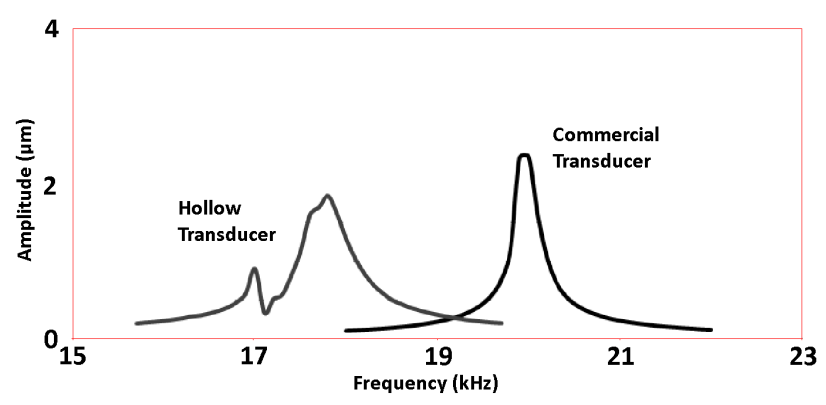

(a)

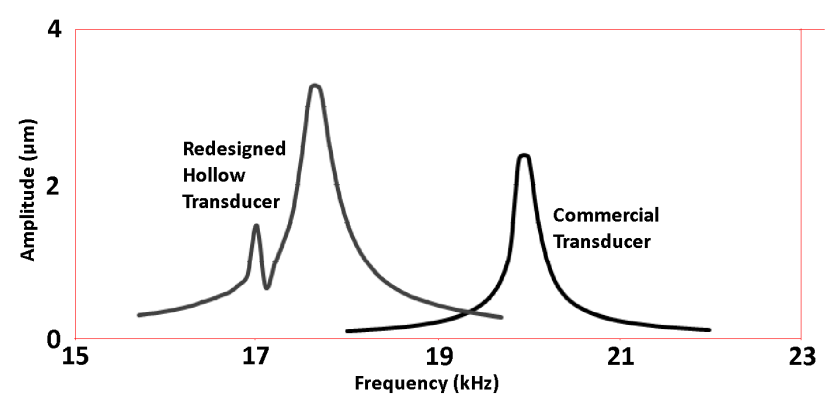

(b)

Figure 4. The response of the hollow transducer (a) before and (b) after the prestress-enhancing modifications, compared to that of a commercial transducer containing the same volume of PZT.

The transducer resonance is $10 \%$ below the design frequency and the presence of a bending mode near the tuned frequency requires careful driving for effective use. However, the additional preload beyond the point of resonance stabilisation increased the amplitude response of the hollow transducer from 1.8 to $3.2 \mu \mathrm{m}$ and increased the Q-factor to a level comparable to that of the commercial transducer.

These disparities are to a large extent the result of high tolerance requirements for the manufacture of the hollow transducer. The wall-thickness of the central bolt is just $3 \mathrm{~mm}$, and so a small 
manufacturing error can have a significant effect on the stiffness of the structure, whereas the same error on a solid bolt has a negligible effect. The hollow transducer is effective in delivering the required ultrasonic amplitude to a coring device and will form the basis of the ongoing design of a completely hollow ultrasonic coring system.

\section{Conclusions}

The design process leading to a prototype of a hollow Langevin transducer has found that the frequency of the operating longitudinal mode increases and tends towards the FE predictions as the mechanical preload is increased, until frequency stabilisation occurs. Thereafter the results indicate that increased preload continues to improve the mechanical quality factor of the transducer and therefore its ultrasonic vibration amplitude.

In terms of mechanical efficiency, the performance of the prototype hollow transducer was comparable to that of a commercial transducer containing the same volume of piezoceramic. The peak output at $40 \mathrm{~V}$ peak-to-peak excitation was over $3 \mu \mathrm{m}$ compared to just over $2 \mu \mathrm{m}$ for the commercial model. However, the Q-factor was significantly lower and an unwanted bending mode close to the main resonance remains a cause for concern.

Technical issues highlighted by the investigation centre on the design of the central bolt. Extremely tight manufacturing tolerances are required to ensure that its mechanical stiffness is correct, and the thread which receives the preload nut should be shallow yet extend over the greatest possible axial distance.

The hollow transducer also requires a long stack of piezoelectric elements if a significant volume of ceramic is to be incorporated. This, coupled with the low stiffness of the hollow bolt, places a constraint on the maximum operating frequency. Unwanted bending modes also appear to be a more significant problem than is the case for traditional solid bolted Langevin transducers. However, the long stack does lend itself to higher ultrasonic vibration amplitudes because equivalent percentage strains translate to higher absolute displacement.

\section{References}

[1] X. Bao, Y. Bar-Cohen, Z. Chang, B. Dolgin, S. Sherrit, D. Pal, S. Du and T. Peterson: IEEE Transactions of UFFC. Vol. 50 (2003), p. 1147

[2] P. Harkness, A. Cardoni and M. Lucas: AIAA 09-6507 (2009)

[3] J. Gallego-Juarez, G. Rodriguez-Corral and L. Gaete-Garreton: Ultrasonics Vol. 16 (1978), p. 267

[4] A. Abdullah, M. Shahini and A. Pak: J. Electroceram. Vol. 22 (2009), p. 369

[5] K. Adachi, M. Tsuji and H. Kato: J. Acoust. Soc. Am. Vol. 105 (1999), p. 1656

[6] T. Takahashi and K. Adachi: Jpn. J. Appl. Phys. Vol. 47 (2008), p. 4736

[7] F. Arnold and S. Muhlen: Ultrasonics Vol. 41 (2003), p. 191

[8] B. Fu, T. Li and Y. Xie: Model-Based Diagnosis for Pre-Stress in Langevin Transducers IEEE Circuits and Systems Technical Conference (2009).

[9] G. Bromfield, U.S. Patent 7627936. (2009).

[10] B. Dubus, G. Haw, C. Granger and O. Ledez: Ultrasonics Vol. 40 (2002), p. 903 